\title{
Artroplastia total de cadera posterior a una infección por Tuberculosis: Caso clínico y revisión de la literatura
}

\section{Total Hip Arthroplasty for the Treatment of Tuberculosis: Case Report and Literature Review}

\author{
Resumen \\ Palabras Clave \\ - artroplastia total de \\ cadera \\ - tuberculosis \\ - artritis séptica \\ tuberculosa
}

Carlos Cabello ${ }^{1}$ César Wilson ${ }^{1}$ Felipe Lopez ${ }^{1}$ Cecilia Mesa $^{2}$

${ }^{1}$ Hospital de Talca, Universidad Católica del Maule, Talca, Chile

${ }^{2}$ Cesfam Dr. Ricardo Valdés, Cauquenes, Chile

Rev Chil Ortop Traumatol 2018;59:82-86.
Address for correspondence César Wilson, MD, Hospital de Talca, Universidad Católica del Maule, Talca, Chile (e-mail: cwilson@hotmail.cl).
La tuberculosis persiste siendo un problema importante a nivel mundial. A pesar de que el compromiso de la cadera es infrecuente, su aparición representa un escenario complejo. Presentamos un paciente masculino que desarrolló una infección de la cadera que fue diagnosticada como tuberculosis de forma tardía. El paciente recibió tratamiento médico por 6 meses y luego se realizó una artroplastia total de cadera en dos tiempos, con éxito. Presentamos el reporte de un caso completo, junto a su seguimiento al aão y una revisión de la literatura sobre el tema.

Tuberculosis remains a significant problem worldwide. Even thought hip involvement is an infrequent manifestation, its appearance represents a challenging scenario. We present a male patient who developed a hip infection later discovered to be tuberculosis. The patient received a medical treatment protocol for 6 months and was subsequently treated with a two stage total hip arthroplasty. We present the full case report with its 1-year outcome and a literature review.

\section{Introducción}

La tuberculosis se mantiene como una enfermedad prevalente a nivel global, e incluso en un mundo desarrollado ante el aumento de trastornos por inmunodeficiencia. La manifestación osteoarticular representa entre el $1 \%$ y el $3 \%$ de todas las infecciones por tuberculosis ${ }^{1}$ y el compromiso de la cadera podría constituir hasta el $15 \%$ de esa manifestación. ${ }^{2,3}$

La forma de presentación larvada, con un compromiso progresivo de la funcionalidad del paciente, asociado a cambios imagenológicos muy inespecíficos, hacen de la tuberculosis un diagnóstico diferencial difícil, ${ }^{4}$ y en la mayoría de los casos, de sospecha tardía. Es así, como en situaciones de destrucción avanzada del hueso y cartílago articular, el tratamiento sistémico local presenta un desafío importante, existiendo controversia con respecto a la longitud del tratamiento antituberculoso y también, sobre las alternativas y el tiempo de la reconstrucción.

Presentamos un reporte de un caso de un paciente con tuberculosis activa de cadera de difícil diagnóstico, que fue tratado de forma exitosa con una artroplastia total de cadera (ATC) en dos tiempos. El paciente fue informado que los datos clínicos, exámenes y la evolución serían revisados y, posteriormente, enviados para publicación, entregándonos su consentimiento.

\section{Caso Clínico}

Paciente de género masculino de 50 años, sin antecedentes mórbidos de importancia, evaluado por un cuadro de 6 meses de evolución de coxalgia derecha de inicio insidioso, de carácter progresivo hasta limitar actividades de la vida diaria. Dolor sin received

March 22, 2018

accepted

July 9,2018
DOI https://doi.org/

10.1055/s-0038-1668498. ISSN $0716-4548$.
Copyright $\odot 2018$ by Thieme Revinter

Publicações Ltda, Rio de Janeiro, Brazil
License terms

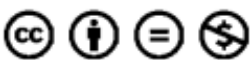


irradiación que cede parcialmente a analgésicos. En su historia destaca la ausencia de traumatismo, fiebre, compromiso del estado general u otros síntomas.

Al momento del examen físico se evidencia impotencia funcional a la marcha. Los rangos articulares se encuentran disminuidos: flexión de $70^{\circ}$, con severa restricción en rotación externa, interna, abducción y aducción. No se evidencia alteración de los tejidos blandos peri articulares. El resto del examen general se encontraba normal.

Se acompaña de exámenes de laboratorio donde destaca hemograma que muestra leucocitos de 12.324 cels $/ \mu \mathrm{L}$, con formula diferenciada normal, PCR $42 \mathrm{mg} / \mathrm{L}$ (referencia normal: $<20 \mathrm{mg} / \mathrm{L}$ ) y VHS $52 \mathrm{~mm} / \mathrm{h}$.

La radiografía de Pelvis Anteroposterior (- Fig. 1) destaca severa disminución del espacio articular y significativa destrucción ósea de la cabeza femoral y del acetábulo. Se solicita una Resonancia magnética que muestra múltiples abscesos periarticulares con importante edema óseo acetabular y en fémur proximal, compatible con una artritis séptica (-Fig. 2).

Para corroborar esa posibilidad, el paciente es sometido a artrocentesis de cadera, bajo fluoroscopía, de la cual no se puede extraer líquido articular para estudio. Se decide realizar artrotomía por abordaje anterior (Smith-Petersen) en la que se evidencia ausencia de líquido articular, sinovial hipertrófica y daño condral severo, enviando muestras para biopsia y cultivo.

Se inicia tratamiento antibiótico empírico (cefazolina y gentamicina), ante la sospecha de una infección piógena. Tras
14 días los resultados de cultivos resultan negativos. El resultado de la biopsia es informado como tejido inflamatorio crónico linfo-histiocitario con granuloma único bien diferenciado no necrotizante. Se complementa estudio con perfil reumatológico básico, el que resulta negativo.

El paciente es dado de alta luego de completar 2 semanas de antibióticos endovenosos, con indicación de antibioterapia oral; presentándose con leves molestias en los primeros controles ambulatorios. Tres meses después evoluciona con aumento del dolor y claudicación, desarrollando fistula a través de cicatriz del abordaje anterior, asociado a progresión radiológica, por lo que es sometido a aseo quirúrgico, con resección del fémur proximal y colocación de espaciador de cemento con antibiótico no articulado (-Fig. 3).

El estudio histológico de la cabeza femoral y tejidos capsulares, revela proceso inflamatorio granulomatoso con células gigantes multinucleadas compatible con tuberculosis. Tinción de Ziehl Neelsen negativa.

Se deriva al equipo de tuberculosis del Hospital, donde se descarta compromiso pulmonar y renal, iniciándose tratamiento según protocolo nacional durante 6 meses. Evolución favorable con cierre de la fistula, logrando normalización de parámetros inflamatorios.

Tras tres meses de terminado el tratamiento antituberculoso se realiza una ATC no cementada con cabeza de cerámica de cuarta generación y vástago full porous coat para fijación metafiso-diafisiaria (-Fig. 4).

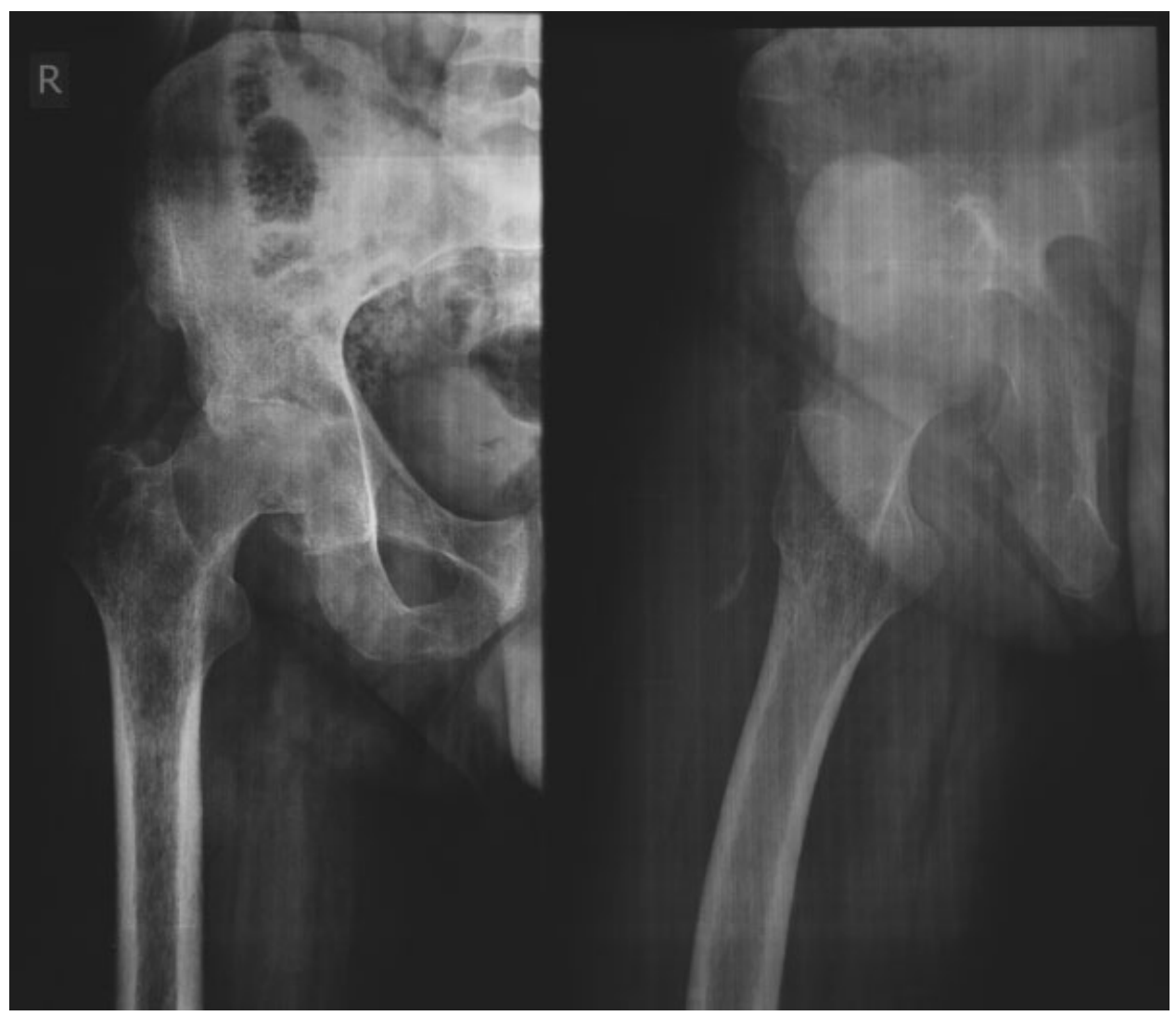

Fig. 1 Rx anteroposterior y axial de cadera derecha. Al momento del ingreso del paciente. Destaca severa disminución del espacio articular. 


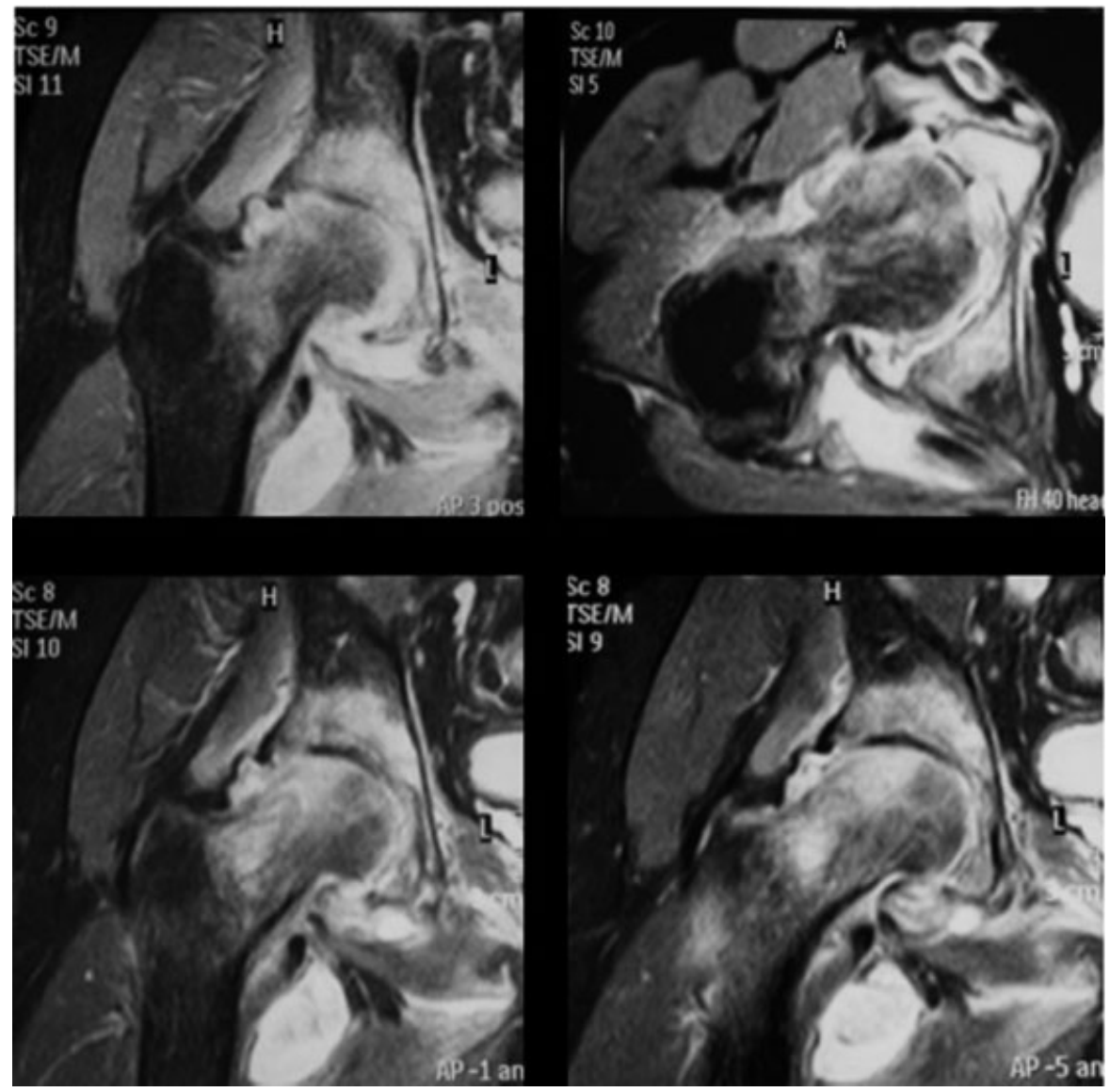

Fig. 2 Secuencia de la RNM. Se evidencia gran derrame articular, deformidad de la cabeza femoral y marcado edema óseo del fémur proximal.

Transcurridos 24 meses de la artroplastia el paciente se encuentra asintomático, mejorando su rango articular y capacidad de marcha, logrando deambular sin bastón, con mínima claudicación. No hay signos de reactivación de la enfermedad.

\section{Discusión}

La tuberculosis y su presentación osteoarticular, a pesar de ser poco frecuente, mantiene su prevalencia a nivel global. El compromiso de la cadera es el segundo más frecuente después de la columna, ${ }^{2}$ y su tratamiento presenta controversias en relación a su manejo médico y quirúrgico. $^{5}$ El caso presentado muestra un paciente con una tuberculosis activa de la cadera que determinó una destrucción articular avanzada. Se describe entonces como se realizó su manejo médico y posterior ATC en 2 tiempos como una alternativa de tratamiento efectiva para ese complejo escenario.

Clásicamente, las opciones de tratamiento quirúrgicas para una TBC de cadera son la artroplastia de resección, artrodesis y ATC. $^{6}$ La artroplastia de resección permite control de la infección, alivio del dolor y aumento del rango articular, pero produce acortamiento de la extremidad, inestabilidad articular y una marcha anormal que generalmente requiere algún tipo de asistencia. ${ }^{7,8}$ La artrodesis, por su parte, disminuye el dolor, estabiliza e inmoviliza la articulación, pero con pobre función de la misma, asociándose a dolor lumbar, gonalgia y marcha asimétrica. Su principal complicación es la no unión, con una incidencia de hasta un $70 \%{ }^{9}$

La ATC se ha establecido como una de las formas más exitosas de tratamiento, con resultados adecuados según seguimientos variables, incluidos 8 a 10 años. ${ }^{1,2}$ Sin embargo, no está claro el momento de realización en pacientes con infección activa o si es necesario esperar a completar el tratamiento de la infección. ${ }^{10}$ Algunos autores recomiendan un largo periodo de quiescencia antes de la cirugía, siendo utilizados intervalos desde ATC inmediata hasta 20 años posterior al inicio de la enfermedad. ${ }^{5,10,11}$

En pacientes con infección activa, el tratamiento quirúrgico con una ATC de forma primaria en un tiempo es controversial por el riesgo potencial de reactivación de la enfermedad. No obstante, la reactivación de la TBC ocurre en pacientes en que no se diagnosticó la etiología inicialmente, en aquellos sin tratamiento preoperatorio o que no presentaron una adherencia adecuada. ${ }^{6}$ Kim y cols., describen 38 pacientes, con periodos de espera que va desde 3 meses a 45 años entre la infección y la artroplastia. Se presentaron 6 casos de reactivación, con resultados funcionales favorables, concluyendo que la artroplastia total es una opción de tratamiento adecuada. ${ }^{11}$

Yoon y cols. ${ }^{10}$ evaluó en forma retrospectiva 7 pacientes con tuberculosis de cadera activa, tratándolos con 


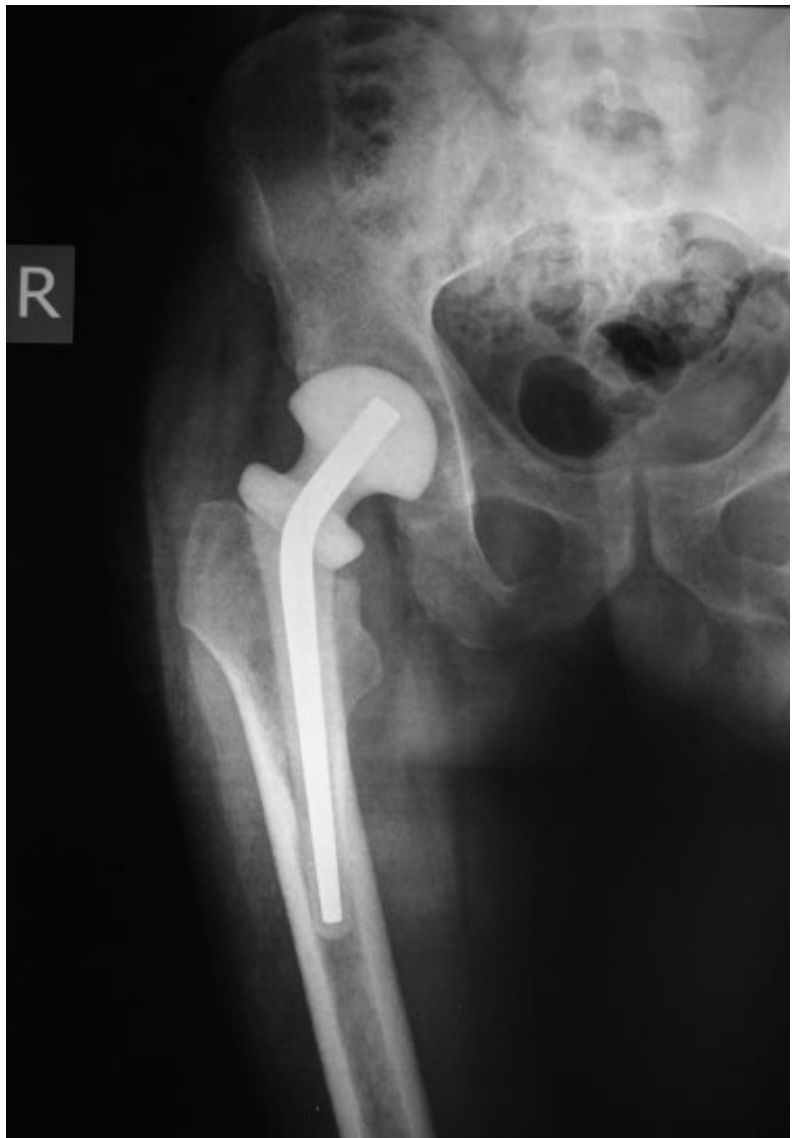

Fig. 3 Rx Posterior a la resección del fémur proximal. Se manejó con un espaciador de cemento para conservar las relaciones anatomicas.

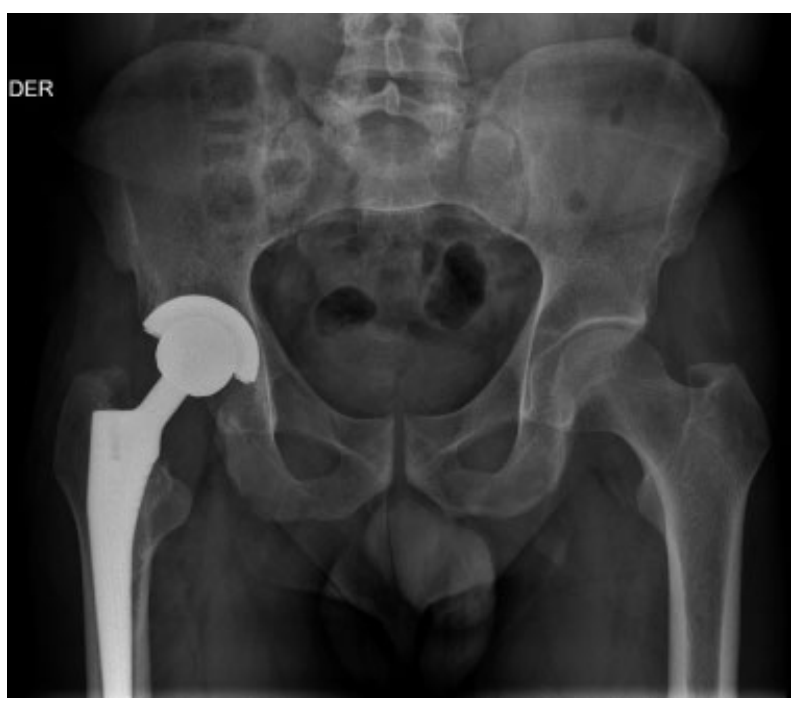

Fig. 4 Rx de pelvis AP. Muestra artroplastia total de cadera no cementada de cadera derecha, cómo resolución del caso.

artroplastia total no cementada, en forma primaria, asociado a curetaje de tejido del tejido inflamatorio, o realizando el reemplazo articular en dos tiempos en caso de existir fistula o de no lograr un debridamiento suficiente, seguido de terapia antituberculosa por 1 año. Obtuvo resultados funcionales favorables sin reactivación. Esto realza el rol de la ATC en la etapa activa de infección, pero de la misma forma, da énfasis a la necesidad de un adecuado debridamiento para obtener buenos resultados.

Se recalca en este trabajo, así como en la mayoría de los autores, la recomendación de realizar la ATC en dos tiempos en el caso de la presencia de trayectos fistulosos o de no lograr un debridamiento adecuado. 2,5,10

El tiempo de duración del tratamiento antituberculoso para el manejo de la tuberculosis extra pulmonar también es tema de debate. Se ha administrado quimioterapia en periodos variables tanto en el pre y postoperatorio, que van desde 2 semanas a tres meses, en el primero, y 6 a 15 meses, en el segundo, sin presentar reactivaciones, mejorando la funcionalidad y obteniendo los mismos resultados que en nuestro paciente. ${ }^{4,10,12,13}$ Es así como se plantea la posibilidad de uso de terapia abreviada, al utilizar un tratamiento asociado con 4 drogas (isoniacida, pirazinamida, rifampicina y etambutol) por 6 meses de la mano de un minucioso debridamiento al momento de realizar el acto quirúrgico. ${ }^{14}$

Wang y cols., reportaron 8 pacientes los cuales fueron sometidos a un tratamiento antituberculoso por 6 meses, previo a la realización de una artroplastia total no cementada. En el seguimiento a 46 meses no se demostró reactivación de la enfermedad, ni falla en los implantes. ${ }^{12}$

En nuestro caso, se presenta un paciente que inicialmente se manejó como artritis séptica, evolucionando de forma tórpida con la formación de fistula a través del abordaje quirúrgico asociado a destrucción acelerada de la articulación. Al igual que lo reportado por Yoon y cols., ${ }^{10}$ nuestro paciente se trató con artroplastia total no cementada, en dos tiempos, debido a la presencia de fistula, manteniéndose con espaciador hasta la cirugía definitiva. ${ }^{5}$ En esa situación, se optó por quimioterapia antituberculosa por una duración de 6 meses, en base a la recomendación a la sociedad americana de enfermedades infecciosas, ${ }^{15}$ mostrando la ausencia de reactivación en el seguimiento de 12 meses posterior a la reconstrucción definitiva.

\section{Conclusión}

La tuberculosis de cadera sigue siendo una entidad poco frecuente, de difícil diagnóstico y que se presenta sin un cuadro clínico característico. Es por eso que debe tenerse presente en todo cuadro de origen incierto o que se aleje de la presentación habitual conocida.

La artroplastia total de cadera es a la fecha, el mejor tratamiento ofrecido para los casos de destrucción avanzada de la articulación, lo que habitualmente es frecuente dado el larvado y difícil diagnóstico. Esa presenta en general buenos resultados, con altos niveles de satisfacción, recuperación de la función y control del dolor. Además, presenta tasas bajas de complicaciones $\mathrm{y}$ reactivaciones, incluso si es realizada en la etapa aguda o en casos en los que la terapia antituberculosa se ha utilizado en periodos abreviados de tratamiento. 


\section{Bibliografía}

1 Tuli SM. General principles of osteoarticular tuberculosis. Clin Orthop Relat Res 2002;(398):11-19

2 Babhulkar S, Pande S. Tuberculosis of the hip. Clin Orthop Relat Res 2002;(398):93-99

3 Garrido G, Gomez-Reino JJ, Fernández-Dapica P, Palenque E, Prieto S. A review of peripheral tuberculous arthritis. Semin Arthritis Rheum 1988;18(02):142-149

4 Wang Y, Wang J, Xu Z, Li Y, Wang H. Total hip arthroplasty for active tuberculosis of the hip. Int Orthop 2010;34(08):1111-1114

5 Li L, Chou K, Deng J, et al. Two-stage total hip arthroplasty for patients with advanced active tuberculosis of the hip. J Orthop Surg 2016;11:38

6 Sidhu AS, Singh AP, Singh AP. Total hip replacement in active advanced tuberculous arthritis. J Bone Joint Surg Br 2009;91(10): 1301-1304

7 Clegg J.The results of the pseudarthrosis after removal of an infected total hip prosthesis. J Bone Joint Surg Br 1977;59(03):298-301

8 Tuli SM, Mukherjee SK. Excision arthroplasty for tuberculous and pyogenic arthritis of the hip. J Bone Joint Surg Br 1981;63-B(01): 29-32
9 Stinchfield FE, Cavallaro WU. Arthrodesis of the hip joint; a follow-up study. J Bone Joint Surg Am 1950;32A(01):48-58

10 Yoon TR, Rowe SM, Santosa SB, Jung ST, Seon JK. Immediate cementless total hip arthroplasty for the treatment of active tuberculosis. J Arthroplasty 2005;20(07):923-926

11 Kim YH, Han DY, Park BM. Total hip arthroplasty for tuberculous coxarthrosis. J Bone Joint Surg Am 1987;69(05):718-727

12 Wang $\mathrm{Q}$, Shen H, Jiang Y, et al. Cementless total hip arthroplasty for the treatment of advanced tuberculosis of the hip. Orthopedics 2011;34(02):90

13 Wang JW. Uncemented total arthroplasty in old quiescent infection of the hip. J Formos Med Assoc 1997;96(08):634-640

14 Neogi DS, Yadav CS, Ashok Kumar, Khan SA, Rastogi S. Total hip arthroplasty in patients with active tuberculosis of the hip with advanced arthritis. Clin Orthop Relat Res 2010;468(02):605-612

15 Blumberg HM, Burman WJ, Chaisson RE, et al; American Thoracic Society, Centers for Disease Control and Prevention and the Infectious Diseases Society. American Thoracic Society/Centers for Disease Control and Prevention/Infectious Diseases Society of America: treatment of tuberculosis. Am J Respir Crit Care Med 2003;167(04):603-662 